\title{
REDUCTION EXPONENT AND DEGREE BOUND FOR THE DEFINING EQUATIONS OF GRADED RINGS
}

\author{
NGÔ VIÊT TRUNG
}

\begin{abstract}
The paper gives upper degree bounds for the defining equations of certain graded rings in terms of the reduction exponent and the multiplicity.
\end{abstract}

1. Introduction. Let $(A, \mathbf{m})$ be a local ring and $\mathbf{a}$ an $\mathbf{m}$-primary ideal. Let $G_{\mathbf{a}}(A)$ denote the associated graded ring $\bigoplus_{n=0}^{\infty} \mathbf{a}^{n} / \mathbf{a}^{n+1}$. Hong [7], Cho [2], and Achilles and Schenzel [1] have shown that if $A$ is a one-dimensional Buchsbaum (resp. Cohen-Macaulay) ring, then the degrees of the defining equations of $G_{\mathbf{a}}(A)$ represented as a quotient of a polynomial ring over $A / \mathbf{a}$ are bounded above by $e(A)+1$ (resp. $e(A)$ ), where $e(A)$ denotes the multiplicity of $A$ with respect to m. The main goal of this paper is to generalize this result for higher-dimensional Buchsbaum (resp. Cohen-Macaulay) rings. Moreover, we also want to explain the phenomenon that all the above works employed, in nonapparent and different ways, the reduction exponent of a minimal reduction of $\mathbf{a}$ and obtained its invariance as a by-product. This is of some interest because Sally [13] has discussed the problem whether $A$ being Cohen-Macaulay implies the invariance of the reduction exponent of minimal reductions of $\mathbf{m}$. Recall that an ideal $\mathbf{b} \subseteq \mathbf{a}$ is called a reduction of $\mathbf{a}$ if $\mathbf{a}^{n+1}=\mathbf{b a}^{n}$ for some nonnegative integer $n[9]$ and that the reduction exponent of $\mathbf{b}$ is the least integer $n$ with this property $[\mathbf{1 1}, \mathbf{1 2}]$. (For the theory of Buchsbaum rings see $[\mathbf{1 4}]$.)

To achieve the above goal we will study the relationship between the reduction exponent $r(I)$ of a minimal reduction $I$ of the positively graded part of a graded ring $R=\bigoplus_{n=0}^{\infty} R_{n}$ and the degrees of the defining equations of $R$ represented as a quotient of a polynomial ring over $R_{0}$. It will turn out that these degrees are bounded above by $\max \{r(I)+1, a(I)\}$, where $a(I)$ denotes the least integer $n$ such that $I$ can be generated by a sequence of elements which is regular in degree $\geq n$. This bound is independent of the choice of $I$ because it can be expressed by means of the local cohomology modules of $R$ in a manner like Castelnuovo's regularity (recently studied by Ooishi [10], and Eisenbud and Goto [4]). If $I$ can be generated by a $d$-sequence [8], we always have $r(I)+1 \geq a(I)$. This implies the invariance of $r(I)$ and the degree bound $r(I)+1$ for the defining equations of a graded Buchsbaum ring $R$ with $R_{0}$ being a zero-dimensional local ring. In particular, by comparing $r(I)$ with the multiplicity $e(R)$ of $R$ with respect to the maximal graded ideal, we shall obtain the following result.

Received by the editors February 7, 1986 and, in revised form, August 14, 1986.

1980 Mathematics Subject Classification (1985 Revision). Primary 13A15; Secondary 13H10.

Key words and phrases. Filter-regular sequence, minimal reduction, reduction exponent, degree bound for the defining equations, Buchsbaum ring.

Part of this paper was done while the author was visiting the University of Genoa by a grant of CNR. He would like to thank both institutions and G. Valla for many inspiring conversations. 
THEOREM 1.1. Let $R$ be a graded Buchsbaum (resp. Cohen-Macauley) ring with $R_{0}$ a zero-dimensional local ring. Then the degrees of the defining equations of $R$ are bounded above by $e(R)+1$ (resp. $e(R))$.

A modified version of the above method applied to $G_{\mathbf{a}}(A)$ will yield the following generalization of the results of Hong, Cho, Achilles, and Schenzel.

THEOREM 1.2. Let $A$ be a Buchsbaum (resp. Cohen-Macaulay) ring with $d=$ $\operatorname{dim}(A) \geq 1$ and $\operatorname{depth}\left(G_{\mathbf{a}}(A)\right) \geq d-1$. Let $r(\mathbf{b})$ denote the reduction exponent of a minimal reduction $\mathbf{b}$ of $\mathbf{a}$. Then

(i) $r(\mathbf{b})$ is independent of the choice of $\mathbf{b}$,

(ii) the degrees of the defining equations of $G_{\mathbf{a}}(A)$ are bounded above by $r(\mathbf{b})+1$,

(iii) $r(\mathbf{b}) \leq e(A) t^{d-1}$ (resp. $e(A) t^{d-1}-1$ ), where $t$ is the least integer with the property $\mathbf{m}^{t} \subseteq \mathbf{a}$.

We would like to mention that a special situation of the Cohen-Macaulay case of Theorem 1.2 was already considered by Cho [2] who claimed that if moreover $G_{\mathbf{a}}(A)$ is free over $A / \mathbf{a}$, the degrees of the defining equation of $G_{\mathbf{a}}(A)$ are bounded above by $e(A)$. But the proof for that contains an error which could not be corrected if $d>1$ (see Remark 5.2).

The proofs of the above theorems will be found in $\S \S 4$ and 5 , respectively. $\S \S 2$ and 3 only have a preparatory character.

2. Filter-regular sequence. Throughout this paper, let $R=\bigoplus_{n=0}^{\infty} R_{n}$ be a noetherian commutative graded ring such that $R_{0}$ is a lock ring and the $R_{0}$-algebra $R$ is generated by the elements of $R_{1}$. Let $M$ denote the unique maximal graded ideal of $R$ and $k$ the residue field $R / M$. Put $R^{+}=\bigoplus_{n \geq 1} R_{n}$. If $E$ is a graded module over $R$, then we denote by $E_{n}$ the $n$th graded piece of $E$.

Let $\mathbf{f}=f_{1}, \ldots, f_{r}$ be a sequence of homogeneous elements of $R, r \geq 1$. One can use the following notion to measure how much $f$ differs from being a regular sequence.

DEFINITION. $\mathbf{f}$ is called $n$-regular if

$$
\left(\left(f_{1}, \ldots, f_{i-1}\right): f_{i}\right)_{n}=\left(f_{1}, \ldots, f_{i-1}\right)_{n}
$$

for $i=1, \ldots, r$. The least integer $m$ such that $\mathbf{f}$ is $n$-regular for all $n \geq m$ will be denoted by $a(\mathbf{f})$.

We shall see that the condition $a(\mathbf{f})<\infty$ can be characterized by the behavior of $f_{i}$ towards the associated primes of $\left(f_{1}, \ldots, f_{i-1}\right), i=1, \ldots, r$.

DEFINITION. f is called filter-regular (with respect ro $R^{+}$) if $f_{i} \notin P$ for all primes $P \in \operatorname{Ass}\left(R /\left(f_{1}, \ldots, f_{i-1}\right)\right), P \nsupseteq R^{+}, i=1, \ldots, r$.

This notion has its origin in the theory of Buchsbaum rings [3] and is closely related to the notion of $d$-sequences [15].

LEMMA 2.1. $a(\mathbf{f})<\infty$ if and only if $\mathbf{f}$ is filter-regular.

PROOF. $\left(\left(f_{1}, \ldots, f_{i-1}\right): f_{i}\right)_{n}=\left(f_{1}, \ldots, f_{i-1}\right)_{n}$ for $n$ sufficiently large if there exists an integer $m$ such that

$$
\left(R^{+}\right)^{m}\left(\left(f_{1}, \ldots, f_{i-1}\right): f_{i}\right) \subseteq\left(f_{1} \ldots, f_{i-1}\right)
$$


or, equivalently,

$$
\left(f_{1}, \ldots, f_{i-1}\right): f_{i} \subseteq \bigcup_{n=0}^{\infty}\left(f_{1}, \ldots, f_{i-1}\right):\left(R^{+}\right)^{n} .
$$

Since $\bigcup_{n=0}^{\infty}\left(f_{1}, \ldots, f_{i-1}\right):\left(R^{+}\right)^{n}$ is the intersection of all primary components of $\left(f_{1}, \ldots, f_{i-1}\right)$ whose associated primes do not contain $R^{+}$, the last inclusion is satisfied iff $f_{i} \notin P$ for all primes $P \in \operatorname{Ass}\left(R /\left(f_{1}, \ldots, f_{i-1}\right)\right), P \nsupseteq R^{+}$.

If $a(\mathbf{f})<\infty$ and $f_{1}, \ldots, f_{r} \in R_{1}, a(\mathbf{f})$ can only take a certain value depending on the number $r$. To see this define

$$
a_{i}(R)=\inf \left\{m \in Z ; H_{R^{+}}^{i}(R)_{n}=0 \text { for all } n \geq m\right\}
$$

for all $i \geq 0$, where $H_{R^{+}}^{i}(R)$ denotes the $i$ th local cohomology module of $R$ with respect to $R^{+}$. For short, we will set $a_{i}=a_{i}(R)$.

PROPOSITION 2.2. Let $\mathbf{f}$ be a filter-regular sequence in $R_{1}$. Then

$$
a(\mathbf{f})=\max \left\{a_{i}+i: i=0, \ldots, r-1\right\} .
$$

We shall need the following lemma in the proof of Proposition 2.2.

LEMMA 2.3. Let $G$ be a filter-regular element in $R_{1}$. Then

$$
a_{i+1}+1 \leq a_{i}(R / g R) \leq \max \left\{a_{1}, a_{i+1}+1\right\}
$$

for all $i \geq 0$.

PROOF. By the definition of a filter-regular sequence, $0: g \subseteq \bigcup_{n=0}^{\infty} 0:\left(R^{+}\right)^{n}$. Therefore, $0: g$ is annihilated by some power of $R^{+}$. Hence $H_{R^{+}}^{i}(0: g)=0$ for $i \geq 1$. Hence from the exact sequence

$$
0 \rightarrow 0: g \rightarrow R \rightarrow R / 0: g \rightarrow 0
$$

we get $H_{R^{+}}^{i}(R)=H_{R^{+}}^{i}(R / 0: g)$ for $i \geq 1$. Now, from the exact sequence

$$
0 \rightarrow R / 0: g \stackrel{g}{\rightarrow} R \rightarrow R / g R \rightarrow 0
$$

one can derive the exact sequence

$$
H_{R^{+}}^{i}(R)_{n} \rightarrow H_{R^{+}}^{i}(R / g R)_{n} \rightarrow H_{R^{+}}^{i+1}(R)_{n-1} \rightarrow H_{R^{+}}^{i+1}(R)_{n}
$$

for $i \geq 0$ which immediately implies the statement.

PROOF OF PROPOSITION 2.2. Since

$$
0: f_{1} \subseteq \bigcup_{n=0}^{\infty} 0:\left(R^{+}\right)^{n} \quad \text { and } \quad f_{1} H_{R^{+}}^{0}(R)_{a_{0}-1} \subseteq H_{R^{+}}^{0}(R)_{a_{0}}=0
$$

$a\left(f_{1}\right)=a_{0}$. Therefore, the case $r=1$ is immediate. For $r>1$ let $\mathbf{f}^{\prime}$ denote the sequence of the images of $f_{2}, \ldots, f_{r}$ in $R / f_{1} R$. By induction and using Lemma 2.3, we have

$$
\begin{aligned}
\max \left\{a_{i}+i ; i=1, \ldots, r-1\right\} & \leq a\left(\mathbf{f}^{\prime}\right)=\max \left\{a_{i}\left(R / f_{1} R\right)+i ; i=0, \ldots, r-2\right\} \\
& \leq \max \left\{a_{i}+i ; i=0, \ldots, r-1\right\} .
\end{aligned}
$$

Thus, since $a(\mathbf{f})=\max \left\{a\left(f_{1}\right), a\left(\mathbf{f}^{\prime}\right)\right\}$, the statement is obvious. 
3. Reduction exponent. Let $\mathbf{n}$ be the maximal ideal of $R_{0}$. By tensoring $R$ with $R_{0}[u]_{(n)}$, where $u$ is some indeterminate, we may always assume that $k$ is infinite. Then there exist minimal reductions of $R^{+}[9]$. Let $I$ be an arbitrary minimal reduction of $R^{+}$. The reduction exponent $r(I)$ is just the least integer $n$ such that $R_{n+1}=I_{n+1}$. The aim of this section is to produce some relationship between $r(I)$ and the local cohomology modules of $R$.

Set $r=\operatorname{dim}(R / \mathbf{n} R)$, the analytic spread of $R^{+}$. It is well known that every homogeneous minimal basis of $I$ consists of $r$ linear forms.

LEMMA 3.1. There exist filter regular sequences of $R$ minimally generating $I$.

ProOF. This statement may be formulated for every homogeneous ideal $J$ with the property $\sqrt{J}=\sqrt{R^{+}}$. Indeed, by induction on $\operatorname{dim}_{k}(J / M J)$, we only need to choose a homogeneous element $g \in J \backslash M J$ such that $g \notin P$ for all primes $P \in \operatorname{Ass}(R), P \nsupseteq R^{+}$. That is always possible because $k$ is infinite and $J \backslash M J$ is not contained in any $P$ by the Nakayama lemma.

PROPOSITION 3.2. $a_{r}+r \leq r(I)+1 \leq \max \left\{a_{i}+i ; i=0, \ldots, r\right\}$.

PROOF. If $r=0, R^{+}$is nilpotent and $r(I)$ is the least integer $n$ such that $R_{n+1}=0$. Therefore, $r(I)+1=a_{0}$ because $H_{R^{+}}^{0}(R)=R$. If $r>1$, choose a filterregular sequence $f_{1}, \ldots, f_{r}$ generating $I$. Note that $I / f_{1} R$ is a minimal reduction of $R^{+} / f_{1} R$ with $r\left(I / f_{1} R\right)=r(I)$. Then the statement will follow from the induction hypothesis on $R / f_{1} R$ by using Lemma 2.3 .

At this point we remark that $\max \left\{a_{i}+i ; i=0, \ldots, r\right\}-1$ is the Castelnuovo regularity of $R[\mathbf{1 0}]$. In particular, if we define

$$
a(I):=\inf \{a(\mathbf{f}) ; I=(\mathbf{f})\}
$$

then we have the following relation.

COROLlaRY 3.3. $\max \{r(I)+1, a(I)\}=\max \left\{a_{i}+i ; i=0, \ldots, r\right\}$.

ProOF. By Lemma 2.1, Proposition 2.2, and Lemma 3.1,

$$
a(I)=\max \left\{a_{i}+i ; i=0, \ldots, r-1\right\} .
$$

Hence the statement follows from Proposition 3.2.

LEMMA 3.4. Suppose that $I$ is minimally generated by a d-sequence $\mathbf{f}$. Then $r(I)+1 \geq a(I)$.

ProOF. By the definition of a $d$-sequence,

$$
\left(\left(f_{1}, \ldots, f_{i-1}\right): f_{i}\right) \cap I=\left(f_{1}, \ldots, f_{i-1}\right)
$$

for $i=1, \ldots, r[\mathbf{8}, \mathbf{1 5}]$. If $n \geq r(I)+1$, then $R_{n}=I_{n}$ and hence

$$
\left(\left(f_{1}, \ldots, f_{i-1}\right): f_{i}\right)_{n}=\left(\left(f_{1}, \ldots, f_{i-1}\right): f_{i}\right)_{n} \cap I_{n}=\left(f_{1}, \ldots, f_{i-1}\right)_{n} .
$$

In the following we call $R$ a graded Buchsbaum ring if $R_{M}$ is a Buchsbaum local ring [14]. 
COROLLARY 3.5. Suppose that $R$ is a graded Buchsbaum ring with $\operatorname{dim}\left(R_{0}\right)=$ 0. Then $r(I)+1=\max \left\{a_{i}+i ; i=0, \ldots, r\right\}$ (independent of the choice of $I$ ).

PROOF. Since $\operatorname{dim}\left(R_{0}\right)=0, I$ can be generated by a homogeneous sytem of parameters of $R$. By [8], $R$ is Buchsbaum iff every stystem of parameters of $R$ is a $d$-sequence. Hence the statement follows from Lemma 3.4.

Corollary 3.5 is a generalization of the formula $r(I)=a_{d}+d-1$ for CohenMacaulay rings $R[\mathbf{5}]$. In general, if $r(I)$ is independent of the choice of $I, r(I)$ need not be $\max \left\{a_{i}+i ; i=0, \ldots, r\right\}-1$.

EXAMPLE. Let $R=k[X, Y] /\left(X^{2}, X Y^{2}\right)$. Then every minimal reduction of $R^{+}$ has the reduction exponent 1 , whereas $a_{0}=3$.

4. Degree bound. Let $f_{1}, \ldots, f_{s}$ be a minimal basis of the $R_{0}$-module $R_{1}$. Then one can define a natural map from the polynomial ring

$$
R_{0}[X]:=R_{0}\left[X_{1}, \ldots, X_{s}\right]
$$

to $R$ by sending $X_{i}$ to $f_{i}, i=1, \ldots, s$. Let $P$ denote the kernel of this map. It is not hard to see that the set of integers occurring as degrees of elements of homogeneous minimal bases of $P$ is independent of the choice of $f_{1}, \ldots, f_{s}$. Let $m_{R}$ denote the maximum of these integers.

PROPOSITION 4.1. $m_{R} \leq \max \{r(I)+1, a(I)\}$ for every minimal reduction $I$ of $R^{+}$.

ProOF. Let $Q$ denote the ideal of all elements of $R_{0}[X]$ whose images in $R$ belong to $I$. Let $m$ denote the maximum degree of elements of all homogeneous minimal bases of $Q$. Since $R_{0}[X]_{n}=Q_{n}$ for $n \geq r(I)+1, m \leq r(I)+1$. Hence we may assume that $m_{R}>m$. Choose $r$ linear forms $F_{1}, \ldots, F_{r}$ such that the sequence $\mathbf{f}$ of their images in $R$ forms a basis of $I$ with $a(\mathbf{f})=a(1)$. Let $F \in P$ be an arbitrary form of degree $m_{R}$. Since $F_{1}, \ldots, F_{r}$ can be extended to a minimal basis of $Q$ by forms of $P, F=G+\sum_{i=1}^{r} F_{i} G_{i}$ for some forms $G$ and $G_{i}$ such that $G$ belongs to the ideal $Q^{\prime}$ generated by forms of $P$ of degree $<m_{R}$. If $m_{R}>a(I)$,

$$
G_{r} \in\left(\left(P, F_{1}, \ldots, F_{r-1}\right): F_{r}\right)_{m_{R}-1}=\left(P, F_{1}, \ldots, F_{r-1}\right)_{m_{R}-1} .
$$

Hence we can omit the term $G_{r} F_{r}$ in the above presentation of $F$. Proceeding like that, we can successively omit $G_{r-1} F_{r-1}, \ldots, G_{1} F_{1}$ and obtain $F \in Q^{\prime}$, a contradiction.

Combining Proposition 4.1 with Corollary 3.3, we get

$$
m_{R} \leq \max \left\{a_{i}+i ; i=0, \ldots, r\right\}
$$

which generalizes a result of Ooishi [10, Proposition 20], and Eisenbud and Goto [4, Theorem 1.2]. In particular, by Corollary 3.5, we have the following consequence.

COROLLARY 4.2. Let $R$ be a graded Buchsbaum ring with $\operatorname{dim}\left(R_{0}\right)=0$. Then $m_{R} \leq r(I)+1$ for any minimal reduction $I$ of $R^{+}$.

PROOF OF THEOREM 1.1. By Corollary 4.2, it suffices to show that $r(I) \leq$ $e(R)$ (resp. $e(R)-1$ ). Since $\operatorname{dim}\left(R_{0}\right)=0, I$ is a minimal reduction of $M$. Hence $e(R)$ is equal to the multiplicity of $R$ with respect to $I$. Note that $I$ is generated by a homogeneous system of parameters $\mathbf{f}=f_{1}, \ldots, f_{r}$ of $R$. If $R$ is Cohen-Macaulay, $r(I) \leq l(R / I)-1=e(R)-1$. If $R$ is Buchsbaum and $r=1$, consider the ring 
$R^{\prime}=R / H_{m}^{0}(R)$. Then $r\left(I R^{\prime}\right) \leq e\left(R^{\prime}\right)-1$ because $R^{\prime}$ is a Cohen-Macaulay ring. Since $R^{+} H_{M}^{0}(R)=0[\mathbf{1 4}]$, it is easy to to check that $r\left(I R^{\prime}\right) \geq r(I)-1$ and $e\left(R^{\prime}\right)=e(R)$. Hence $r(I) \leq e(R)$. If $r>1$, consider the $\operatorname{ring} R / f_{1} R$. Then $r\left(I / f_{1} R\right)=r(I)$ and by the definition of Buchsbaum rings, $e\left(R / f_{1} R\right)=e(R)$. Hence $r(I) \leq e(R)$ by the induction hypothesis of $R / f_{1} R$.

It is worth noticing that the bound $r(I)+1$ of Corollary 4.2 is much better than the bound $e(R)+1$ of Theorem 1.1.

EXAMPLE. Let $R$ be the homogeneous coordinate ring of a projective curve given parametrically by a set $S$ of monomials of degree $e$ in two indeterminates $t, s$ such that $r^{e}, t^{e-1} s, t s^{e-1}, s^{e} \in S$. Consider $R$ as the subring of $k[t, s]$ generated by the monomials of $S$. Put $I=\left(t^{e}, s^{e}\right)$. Then $r(I) \leq 2$ if $R$ is a Buchsbaum ring [16, Theorem 4.1], whereas $e(R)=e$ may be arbitrarily large. A famous example is the twisted cubic curve with the parameters $t^{4}, t^{3} s, t s^{3}, s^{4}$ which is known to be arithmetically Buchsbaum.

If $R_{0}=k$, Ooishi [10, Theorems 19 and 20] has given the bound $m_{R} \leq e(R)+$ $r-\operatorname{dim}\left(R_{1}\right)+i(R)+1$, where $i(R):=\sum_{i=0}^{r-1}\left(\begin{array}{c}r-1 \\ i\end{array}\right) H_{M}^{i}(R)$. Except in the case where $R$ is a Cohen-Macaulay ring, this bound is not better than the bound $e(R)+1$ of Theorem 1.1.

EXAMPLE. Let $R$ be as in the above example with $S=\left\{t^{3 p-i+1} s^{i} ; i=0,1,3 p\right.$, $3 p+1$ and $i=3 j-1$ with $j=1, \ldots, p\}$, where $p$ is some positive integer. By [16, Theorem 4.1], it is easy to check that $R$ is a Buchsbaum ring with $i(R)=2 p-2$. Therefore $e(R)+1=3 p+2<4 p-2=e(R)+r-\operatorname{dim}\left(R_{1}\right)+i(R)+1$ if $p>4$.

5. The local case. Let a be an arbitrary ideal of the local ring $(A, \mathbf{m})$. Set $r=\operatorname{dim}\left(G_{\mathbf{a}}(A) / \mathbf{m} G_{\mathbf{a}}(A)\right)$, the analytic spread of $\mathbf{a}$. Then a is called equimultiple if ht $(\mathbf{a})=r[6]$. This notion has some relation to Zariski's equimultiplicity and is satisfied if $A$ is normal flat along a (an important notion in Hironaka's resolution of singularities). The class of equimultiple ideals is rather large. It contains e.g. all m-primary ideals.

Proposition 5.1. Let $A$ be a Buchsbaum ring and a an equimultiple ideal. Let $x_{1}, \ldots, x_{r}$ be elements of $\mathbf{a}$ such that $\mathbf{b}=\left(x_{1}, \ldots, x_{r}\right)$ is a minimal reduction of a and

$$
\left(x_{1}, \ldots, x_{i}\right) \cap \mathbf{a}^{n+1}=\left(x+1, \ldots, x_{i}\right) \mathbf{a}^{n}
$$

for all $n \geq 0, i=1, \ldots, r-1$. Let $r(\mathbf{b})$ denote the reduction exponent of $\mathbf{b}$. Set $R=G_{\mathbf{a}}(A)$. Then

(i) $r(\mathbf{b})=\max \left\{a_{i}+i ; i=0, \ldots, r\right\}-1$,

(ii) $m_{R} \leq r(\mathbf{b})+1$.

PROOF. Let $f_{1}, \ldots, f_{r}$ be the initial forms of $x_{1}, \ldots, x_{r}$ in $R$. Then $I=$ $\left(f_{1}, \ldots, f_{r}\right)$ is a minimal reduction of $R^{+}$with $r(I)=r(\mathbf{b})$. By Corollary 3.3 and Proposition 4.1 , it suffices to show that $r(I)+1 \geq a(\mathbf{f})$, i.e. $\left(\left(f_{1}, \ldots, f_{i-1}\right): f_{i}\right)_{n}=$ $\left(f_{1}, \ldots, f_{i-1}\right)_{n}$ for $n \geq r(I)+1, i=1, \ldots, r$. Translating this condition in terms of $x_{1}, \ldots, x_{r}$, we have to show that

$$
\left[\left(\left(x_{1}, \ldots, x_{i-1}\right) \mathbf{a}^{n}+\mathbf{a}^{n+2}\right): x_{i}\right] \cap \mathbf{a}^{n}=\left(x_{1}, \ldots, x_{i-1}\right) \mathbf{a}^{n-1}+\mathbf{a}^{n+1} .
$$

Since the left side obviously contains the right side, it is sufficient to prove the converse inclusion. Let $x$ be an arbitrary element of the left side. Then $x_{i} x=y$ modulo $\left(x_{1}, \ldots, x_{i-1}\right) \mathbf{a}^{n}$ for some element $y \in\left(x_{1}, \ldots, x_{i}\right) \cap \mathbf{a}^{n+2}=\left(x_{1}, \ldots, x_{i-1}\right) \mathbf{a}^{n+1}$. 
Thus, there is an element $z \in \mathbf{a}^{n+1}$ such that $x_{i}(x-z) \in\left(x_{1}, \ldots, x_{i-1}\right)$. Since $\mathrm{ht}(\mathbf{b})=\operatorname{ht}(\mathbf{a})=r, x_{1}, \ldots, x_{r}$ belong to a system of parameters of $A$. Therefore, $x_{1}, \ldots, x_{r}$ is a $d$-sequence [8]. Hence $\left(\left(x_{1}, \ldots, x_{i-1}\right): x_{i}\right) \cap \mathbf{b}=\left(x_{1}, \ldots, x_{i-1}\right)$. Note that $\mathbf{a}^{n}=\mathbf{b a}^{n-1} \subseteq \mathbf{b}$. Then

$$
\begin{aligned}
x-z & \in\left(\left(x_{1}, \ldots, x_{i-1}\right): x_{i}\right) \cap \mathbf{a}^{n}=\left(x_{1}, \ldots, x_{i-1}\right) \cap \mathbf{a}^{n} \\
& =\left(x_{1}, \ldots, x_{i-1}\right) \mathbf{a}^{n-1} .
\end{aligned}
$$

Hence we can conclude that $x \in\left(x_{1}, \ldots, x_{i-1}\right) \mathbf{a}^{n-1}+\mathbf{a}^{n+1}$.

Now we will apply Proposition 5.1 to prove Theorem 1.2.

PROOF OF THEOREM 1.2. Without restriction, we may assume that $k=A / \mathbf{m}$ is infinite. Since $\mathbf{a}$ is an $\mathbf{m}$-primary ideal, $\mathbf{b}$ is generated by a system of parameters $x_{1}, \ldots, x_{d}$ of $A$. Set $R=G_{\mathbf{a}}(A)$. By Lemma 3.1, we may assume that the initial forms $f_{1}, \ldots, f_{d}$ of $x_{1}, \ldots, x_{d}$ in $R$ form a filter-regular sequence. Since $\operatorname{depth}(R) \geq$ $d-1, f_{1}, \ldots, f_{d-1}$ is a regular sequence. By $[\mathbf{1 7}]$, this implies the relation

$$
\left(x_{1}, \ldots, x_{i-1}\right) \cap \mathbf{a}^{n+1}=\left(x_{1}, \ldots, x_{i-1}\right) \mathbf{a}^{n}
$$

for all $n \geq 0, i=1, \ldots, d-1$. Hence (i) and (ii) follow from Lemma 5.1. For (iii) we first note that the case $d=1$ is already known [1, Propositions 2.2 and 2.3]. If $d>1, R / f_{1} R=g_{\mathrm{a} / x_{1} A}\left(A / x_{1} A\right)$ because $f_{1}$ is a non-zero-divisor of $R$. Thus, $\operatorname{depth}\left(G_{\mathbf{a} / x_{1} A}\left(A / x_{1} A\right)\right) \geq d-2$ and $r\left(\mathbf{b} / x_{1} A\right)=r\left(I / f_{1} R\right)=r(I)=r(\mathbf{b})$, where $I=\left(f_{1}, \ldots, f_{d}\right)$. By the induction hypothesis,

$$
r(\mathbf{b}) \leq e\left(A / x_{1} A\right) t^{d-2} \quad\left(\text { resp. } e\left(A / x_{1} A\right) t^{d-2}-1\right) .
$$

On the other hand, like in [11, Lemma 1.1], we can choose $x_{1}$ so that the initial form $g$ of $x_{1}$ in $G_{\mathbf{m}}(A)$ is filter-regular. From this it follows that $(0: g)_{n}=0$ for $n$ sufficiently large. Let $s$ be the degree of $g$ in $G_{\mathbf{m}}(A)$. Then $\left(\mathbf{m}^{n+s+1}: x_{1}\right) \cap \mathbf{m}^{n}=$ $\mathbf{m}^{n+1}$ for $n$ sufficiently large, too. Therefore, $x_{1}$ is a superficial element of order $s$ for $\mathbf{m}$ and we get $e\left(A / x_{1} A\right)=e(A) s\left[\mathbf{1 9}\right.$, Chapter VIII, §8]. Since $x_{1} \notin \mathbf{m a} \subseteq \mathbf{m}^{t+1}$, $s \leq t$. Hence we can conclude that

$$
r(\mathbf{b}) \leq e(A) t^{d-1} \quad\left(\text { resp. } e(A) t^{d-1}-1\right) .
$$

REMARK 5.2. In [2, Theorem 2], Cho claimed that if $\mathbf{a}$ is an $\mathbf{m}$-primary ideal of a Cohen-Macaulay ring $A$ such that $R=G_{\mathbf{a}}(A)$ is free over $A / \mathbf{a}$ and $\operatorname{depth}(R) \geq d-1$, then $m_{R} \leq e(A)$. But his proof for the case $d>1$ contains the wrong argument that there is an element $x_{1} \in \mathbf{a}$ such that $e\left(A / x_{1} A\right)=e(A)$. For example, that is impossible if $\mathbf{a} \subseteq \mathbf{m}^{2}$. However, the author of the present paper could neither give another proof nor find a counterexample to this claim. The condition $G_{\mathbf{a}}(A)$ being free over $A$ /a seems to be very strong. For example, if $A$ is regular, this condition implies that $\mathbf{a}$ is generated by a regular sequence [18].

\section{REFERENCES}

1. R. Achilles and P. Schenzel, $A$ degree bound for the defining equation of one-dimensional tangent cones, Seminar D. Eisenbud, B. Singh, W. Vogel, vol. 2, Teubner-Texte zur Math. 48, Leipzig, 1982.

2. Y. H. Cho, Presentation of associated graded rings of Cohen-Macaulay rings, Proc. Amer. Math. Soc. 89 (1983), 569-573.

3. N. T. Cuong, P. Schenzel, and N. V. Trung, Verallgemeinerte Cohen-Macaulay-Moduln, Math. Nachr. 88 (1978), 57-73. 
4. D. Eisenbud and S. Goto, Linear free resolutions and minimal multiplicity, J. Algebra 88 (1984), 89-133.

5. S. Goto and Y. Shimoda, On the Rees algebras of Cohen-Macaulay rings, Commutative Algebra (Fairfax 1979), Lecture Notes in Pure and Appl. Math., vol. 68, Dekker, New York, 1982.

6. U. Grothe, M. Herrmann, and U. Orbanz, Graded Cohen-Macaulay rings associated to equimultiple ideals, Math. Z. 186 (1984), 531-556.

7. Y. H. Hong, Presentation of associated graded rings as quotient of polynomials, Preprint, Northwestern University, Evanston, Ill., 1981.

8. C. Huneke, The theory of d-sequences and powers of ideals, Adv. in Math. 46 (1982), 627-644.

9. D. G. Northcott and D. Rees, Reductions of ideals in local rings, Proc. Cambridge Philos. Soc. 50 (1954), 145-158.

10. A. Ooishi, Castelnuovo's regularity of graded rings and modules, Hiroshima Math. J. 12 (1982), 627-644.

11. J. Saly, Bounds for numbers of generators of Cohen-Macaulay rings, Pacific J. Math. 63 (1976), 517-520.

12. __ Tangent cones at Gorenstein singularities, Compositio Math. 40 (1980), 167-175.

13. _ Reductions, local cohomology, and Hilbert functions of local rings, Commutative Algebra (Durham 1981), London Math. Soc. Lecture Note Series, no. 72, Cambridge Univ. Press, 1982.

14. J. Stückrad and W. Vogel, Toward a theory of Buchsbaum singularities, Amer. J. Math. 100 (1978), 727-746.

15. N. V. Trung, Absolutely superficial sequences, Math. Proc. Cambridge Philos. Soc. 93 (1983), 35-47.

16. __ Projections of one-dimensional Veronese varieties, Math. Nachr. 118 (1984), 47-67.

17. P. Valabrega and G. Vaala, Form rings and regular sequences, Nagoya Math. J. 72 (1978), 93-101.

18. W. Vasconcelos, Ideals generated by $R$-sequences, J. Algebra 6 (1967), 309-316.

19. O. Zariski and P. Samuel, Commutative algebra. II, Springer-Verlag, Berlin and New York, 1975.

Institute of Mathematics, BoX 631, Bò Hô, Hanoi, VietnaM 